\title{
GRAVITATIONAL COLLAPSE OF MASS-ACCRETING WHITE DWARFS
}

\author{
J. Isern', F. Canal2, D. García ${ }^{3}$, M. Hernanz ${ }^{3}$, and J. Labay \\ ${ }^{1}$ Centre d'Estudis Avançats de Blanes, CSIC \\ 17300 Blanes (Girona), Spain \\ 'Departament de Física de 1 'Atmosfera, Astronomia i Astrofísica \\ Universitat de Barcelona, 08028 Barcelona, Spain \\ Universitat Folitecnica de Catalunya \\ o802日 Barcelona, Spain
}

\section{INTEODUCTION}

Massive star ( $M \geq 10 M$ ) core collapse is the standard mechanism for neutron star formation (see Brown 1988 for a recent review). It has long been realized (see, for instance, van den Heuvel 1988 , and references therein) that the neutron 5 tars found in different types of binary systems cannot come from such a standard mechanism. Those systems include wide binary radio pulsars, millisecond pulsars (not in wide binaries), galactic bulge X-ray sources (including GPO's), type I $x-r a y$ burst sources and $x-r a y$ transients, and $\gamma-r a y$ sources. Formation of those neutron stars is now widely attributed to the gravitational collapse of a white dwarf, growing above Chandrasekhar's 1 imit by mass accretion from the current neutron star's companion in the binary system Canal and Schatzman 1776; Canal and Isern 1979; Canal, Isern, and Labay 1980; Miyaji et al. 1980). Mass growth up to dynamical instability means that both explosive ejection of the accreted layers and explosive disruption of the whole star must be avoided. The former is associated with the nova phenomenon. The latter, with the occurrence of type I supernovae.

Concerning nova outbursts, the results as to the ranges of different parameters (initial mass and temperature of the star, mass accretion rate, chemical composition of both the star and the accreted material, allowing mass growth are so divergent, when the simplest hypotheses (spherical symmetry, no kinetic energy nor angular momentum deposition) are relaxed (see Sparks and Kutter 1987; Shaviv and Starrfield 1997) that those parameters should presently be regarded as almost free. Explosive ignition of electron-degenerate c+o cores has been studied by Nomoto, Thielemann, and Yokoi (1984), and Sutherland and wheeler (1984). Ignition densities were in the range $\rho \cong(2-4) \times 10^{\circ}$ $9 \mathrm{~cm}^{-9}$ and the cores were entirely fluid. Hydrodynamic burning 
propagation leads to their complete disruption. Ignition at higher densities happens when the cores are cold and massive enough at the start of mass accretion (Hernanz et al. 1988). The range studied

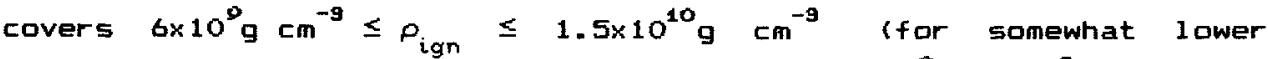
initial masses it covers the whole interval $2 \times 10^{\circ} \mathrm{g} \mathrm{cm}-\mathrm{a} \leq \rho_{\text {ign }} \leq$ $1.5 \times 10^{10} \mathrm{~g} \mathrm{~cm}^{-3}$, and carbon ignition takes place in solid layers when $\rho_{\text {ign }} \geq 9.5 \times 10^{\circ} \mathrm{g} \mathrm{cm}^{-3}$. Ignition would be delayed up to still higher densities if carbon and oxygen were inmiscible in solid phase (Stevenson 1980; Labay et al. 1985). Here we try to determine the critical ignition density corresponding to bifurcation between stellar explosion (either leading to complete disruption or leaving some white dwarf remnant) and gravitational collapse leading to neutron star formation and we analyze the physical processes involved. $0+N e+M g$ cores have also been proposed as progenitors of neutron stars (Miyaji et al. 1980). Here we will briefly outline the uncertainties still involved as to their behaviour upon mass accretion.

\section{DEGENERATE CARBON IGNITION AT VERY HIGH DENSITIES}

Initially massive $\left(M \geq 1.2 \mathrm{M}\right.$ ) and cold $\left(T \leq 5 \times 10^{7} \mathrm{~K}\right) \mathrm{C}+\mathrm{T}$ white dwarfs do ignite their thermonuclear fuel at higher densities than both less massive and hotter white dwarfs and intermediate mass 84 M $\leq M \leq 8 M$ ) red giant cores (see Hernanz et al. 1988, and references therein). This is due to the fact that pycnonuclear reaction rates (in the solid phase) are much lower than strongly screened thermonuclear rates (in the fluid phase) for C+D mixtures at a given density. Higher ignition densities also mean higher electron capture rates on the incinerated (NSE) material after thermonuclear runaway. When the surrounding layers are still solid at central carbon ignition, this equally means conductive burning propagation, typically at velocities of the order of 0.01 times the local velocity of sound $c_{s}$ (it must be noted that conduction will dominate anyway close to the star's center, even in fluid layers: see woosley and weaver 1986). Both facts do favour electron captures (driving contraction and potentially leading to gravitational collapse) against thermonuclear burning propagation (driving hydrodynamical expansion, with potential disruption of the star). Solidification of a central core prior to the mass-accretion stage in the white dwarf's evolution would produce still more drastical effects if it were to lead to chemical separation of oxygen from carbon (Stevenson 1980; Mochkovitch 1993). Recent calculations by Barrat, Hansen, and Mochkovitch (1988) do indicate that carbon and oxygen are miscible in solid phase inonetheless, these calculations 
still involving fairly arbitrary approximations, chemical separation is not yet completely ruled out).

In previous papers CCanal and Isern 1979; Isern, Labay, and Canal 1984; Isern et al. 1988) we have already addressed the question as to the critical ignition density for collapse of a C+D white dwarf into a neutron star and its dependence on the still uncertain physics of thermonuclear burning propagation. In Table 1 we summarize the results from several calculations based on two deflagrating models taken from Hernanz et al. (1988).

TABLE 1

\begin{tabular}{|c|c|c|c|c|}
\hline Model & $\left(g \mathrm{~cm}^{-9}\right)$ & $v_{\text {burn }} / c_{s}$ & Outcome & $t_{11}$ \\
\hline$A$ & $9.50 \times 10^{\circ}$ & 0.005 & Coll apse & 1.47 \\
\hline E. & $1.09 \times 10^{10}$ & 0.005 & Coll apse & 1.09 \\
\hline $\mathbf{B}$ & $1.09 \times 10^{10}$ & 0.010 & Coll apse & 0.93 \\
\hline $\mathbf{E}$ & $1.09 \times 10^{10}$ & 0.100 & Explosion & -- \\
\hline
\end{tabular}

Burning front velocities in the two first rows of Table 1 are average values when using Woosley and Weaver"s (1986) expression for conductive velocities. The other two values correspond to parametrization of burning propagation speed. $t_{11} i s$ the $t i m e$ elapsed between explosive ignition (at the indicated densities) and contraction to a central density of $10^{11} \mathrm{~g} \mathrm{~cm}^{-9}$. When "collapse" is indicated as the outcome, the star is homologously contracting on a hydrodynamical time scale and its mass (due to electron captures) is above Chandrasekhar's mass. We see that, for ignitions at densities of the order of $10^{10} \mathrm{~g} \mathrm{~cm}^{-3}$, the bifurcation between collapse and explosion is located between 0.01 and 0.1 times the local sound velocity. Concerning densities, for conductive burning, the minimum value for collapse is around $9.5 \times 10^{\circ} \mathrm{g} \mathrm{cm}^{-3}$. Comparison of Table 1 with Nomoto's (1986, 1987) recent results shows very big discrepancies. These are probably due to some mistake in the calculation of the burned mass (see, for instance, Nomoto's case D).

\section{DEGENERATE QXYGEN IGNITION}

O+Ne+Mg white dwarfs are also candidates to gravitational collapse upon mass accretion (Miyaji et al. 1980). Dxygen ignition is 
triggered by electron captures on ${ }^{20} \mathrm{Ne}$. But the exact density at which this happens depends on the adopted criterion for convective instability and on the treatment of semiconvection (Mochkovitch 1984; Miyaji and Nomoto 1987). Ignitian at $\rho_{\text {ign }} \leq 9.5 \times 10^{\circ} \mathrm{g} \mathrm{cm}^{-3}$ is 1 ikely and the outcome (collapse or explosion) is still uncertain.

\section{REFERENCES}

Barrat, J.L., Hansen, J.P., Mochkovitch, R. 1988, Astron. Astrophys., 199, L. 15

Brown, G.E. 1988, Phys. Rept., 163, 1

Canal, R., Schatzman, E. 1976, Astron. Astrophys, 46, 229

Canal, R., Isern, J. 1979, in white Dwarfs and Variable Deqenerate Stars, ed. H.M. Van Horn and $V$. Weidemann Univ. Rochester Fress, Rochester), 0.52

Canal, R., Isern, J., Labay, J. 1980, Astrophys. J. (Letters), 241, L33

Hernanz, M., Isern, J., Canal, R., Labay, J., Mochkovitch, Fi. 1989, Astrophys. J., 324, 331

Isern, J., Labay, J., Canal, Fi. 1984, Nature, 309, 431

Isern, J., Canal, R., Garcia, D., Garcia-Berro, E., Hernanz, M., Labay, J. 1988, AdV. Space Res., in the press

Labay, J., Canal, R., Garcia-Berro, E., Hernanz, M., Isern, J. 1985, in Fiecent Results on Cataclysmic Variables, ed. J. Fahe (ESA 5P-236)

Miyaji, S., Nomoto, K., Yokoi, K., Sugimoto, D. 1980, Fub. Astron. Soc. Japan, 32, 303

Miyaji, 5., Nomoto, K. 1987, Astrophys. J., 318, 307

Mochkavitch, R. 1983, Astron. Astrophys., 122, 212

Nomoto, K. 1986, Progr. Fart. Nucl. Phys., 17, 249

Nomoto, K. 1987, in The Origin and Evolution of Neutron Stars, ed. D.J. Helfand and J. - H. Huang (Feidel, Dordrecht), $P .281$

Nomoto, K., Thielemann, F.K., Yokai, K. 1984, Astrophys. J., 286, 644

Shaviv, G., Starrfield, S. 1987, Astrophys. J. (Letters), 321, L51

Sparks, W.M., Kutter, G.S. 1987 Astrophys. J., 321, 394

Stevenson, D.J. 1980, J. Phys. Suppl., No. 3, 41, C2-53

Sutherland, P.G., Wheeler, J.C., 1984, Astrophys. J., 280, 282

Van den Heuvel, E.P.J. 1988, Adv. Space Res., in the press

Wonsley, S.E., Weaver, T.A. 1986, in Radiation Transport and Hydrodynamics, IAU Coll. No. 89, ed. D. Mihalas, K.H. Winkler. Dordrecht: Reidel 